Journal Club

Editor's Note: These short reviews of recent JNeurosci articles, written exclusively by students or postdoctoral fellows, summarize the important findings of the paper and provide additional insight and commentary. If the authors of the highlighted article have written a response to the Journal Club, the response can be found by viewing the Journal Club at www.jneurosci.org. For more information on the format, review process, and purpose of Journal Club articles, please see http://www.jneurosci.org/content/ jneurosci-journal-club.

\title{
Trans-thalamic Pathways: Strong Candidates for Supporting Communication between Functionally Distinct Cortical Areas
}

\author{
-Barna Zajzon ${ }^{1,2}$ and $\oplus^{-A i t o r ~ M o r a l e s-G r e g o r i o ~}{ }^{1,3}$ \\ ${ }^{1}$ Institute of Neuroscience and Medicine (INM-6), Computational and Systems Neuroscience and Institute for Advanced Simulation (IAS-6), Theoretical \\ Neuroscience and JARA-Institut Brain Structure-Function Relationships (INM-10) 52428, ${ }^{2}$ Department of Psychiatry, Psychotherapy and Psychosomatics, \\ RWTH Aachen University, Germany 52074, and ${ }^{3}$ RWTH Aachen University, Germany 52074 \\ Review of Mo and Sherman.
}

\section{Introduction}

The thalamus was long considered a passive relay of sensory information with little or no active role in higher cognitive functions. However, mounting evidence suggests that thalamic nuclei form complex loops with the cortex and are involved in a myriad of cognitive processes, including attention and working memory (Ward, 2013). Although first-order thalamic nuclei (e.g., lateral geniculate nucleus) play a key role in the transmission of ascending sensory input to the cortex, higher-order nuclei (e.g., pulvinar or mediodorsal nucleus) are believed to be involved in sustaining and modulating communication within and between cortical regions (Guillery, 1995). Understanding the functional role of such nuclei in a mechanistic manner requires, in addition to behavioral experiments, a de-

\footnotetext{
Received March 22, 2019; revised June 14, 2019; accepted July 5, 2019.

This work was supported by the European Union's Horizon 2020 Framework Programme for Research and Innovation (Grant 785907, Human Brain Project SGA2), the Priority Program (SPP 2041 "Computational Connectomics") of the Deutsche Forschungsgemeinschaft (S.J. van Albada: AL 2041/1-1), and the Helmholtz Alliance through the Initiative and Networking Fund of the Helmholtz Association. We thank Drs. Renato Duarte and Sacha van Albada for supportive comments and constructive suggestions.

The authors declare no competing financial interests.

Correspondence should be addressed to Barna Zajzon at b.zajzon@fz-juelich.de.

https://doi.org/10.1523/JNEUROSCI.0656-19.2019

Copyright $\odot 2019$ the authors
}

tailed anatomical and physiological mapping of the thalamocortical circuitry.

Thalamic cells in higher-order nuclei have been shown to act as an intermediary between cortical areas, providing a cortico-thalamo-cortical pathway that augments direct communication (Sherman and Guillery, 2013). These circuits are known to originate in layer 5 (L5) of cortex, but their target layers have not been exhaustively studied. They are typically arranged in parallel to strong feedforward corticocortical projections, which in mice usually originate in cortical layers L2/3 and L5 and project to most layers in the target area (e.g., S1 $\rightarrow \mathrm{M} 1$; Porter and White, 1983). Such transthalamic connections involve class 1 or "driver" glutamatergic synapses (Sherman and Guillery, 2011), which produce large, depressing postsynaptic currents, activate mainly ionotropic receptors, and have large and small boutons targeting proximal dendrites. Because of their high probability of neurotransmitter release, class 1 terminals are thought to be efficient information carriers and can reliably elicit thalamic action potentials (Rovó et al., 2012).

Instead of just passively relaying signals from one cortical area to another, the thalamus might manipulate information arriving from L5 in a context-dependent manner. Specifically, thalamic nuclei have been suggested to dynamically construct task-relevant functional circuits (Nakajima and Halassa, 2017), as well as change the effective connectivity between cortical regions through targeted gain modulation (Jaramillo et al., 2019). However, anatomical evidence of such trans-thalamic pathways was previously limited to projections between primary and secondary auditory, visual, and somatosensory cortices (Theyel et al., 2010; Sherman and Guillery, 2013). Given that most higherorder nuclei receive connections from and project to multiple cortical areas, a natural question is whether the existence of parallel routes is a general organizing principle, possibly linking functionally distinct cortical areas.

To answer this question, Mo and Sherman (2019) used trans-synaptic viral tracing in mouse slice preparations to demonstrate the existence of an indirect pathway between the primary somatosensory cortex (S1) and the primary motor cortex (M1) through the thalamic posterior medial (POm) nucleus, a higherorder nucleus in the rodent whisker system that is actively involved in processing sensorimotor information (Krieger and Groh, 2015). Specifically, the authors found M1-projecting POm neurons receiving input from S1 (45.3\%) and S2 (26.3\%). Although neurons projecting to POm were more abundant in L6 of S1, the 
hypothesized projections from L5 of S1 were also found. This establishes the presence of a previously unknown S1 L5 $\rightarrow$ $\mathrm{POm} \rightarrow \mathrm{M} 1$ pathway involving direct monosynaptic connections.

To determine the synaptic properties of the circuit, first the inputs to POm from L5 of S1 were targeted for optogenetic and electrical stimulation. Whole-cell patch recordings of M1-projecting POm neurons revealed large postsynaptic currents and strong paired-pulse depression after both axonal and dendritic stimulation of S1 L5 inputs to POm cells, which were eliminated after blocking ionotropic glutamate receptors. Additionally, bouton size analysis revealed the presence of large and small terminals. As noted above, these characteristics are indicative of class 1 synapses (Sherman and Guillery, 2013). Because the synapses from POm to M1 displayed similar features, the authors concluded that the $\mathrm{S} 1 \mathrm{~L} 5 \rightarrow \mathrm{POm} \rightarrow \mathrm{M} 1$ pathway includes exclusively class 1 synapses. These results are consistent with previous reports (Reichova and Sherman, 2004) and suggest a robust trans-thalamic pathway through POm with synapses well suited for the successful relay of somatosensory information (Sherman and Guillery, 2011).

It should be noted that the net effect of S1 L5 activity on M1 via the POm remains somewhat unclear because the optogenetic stimulation did not exclusively target these projections. Indeed, only onethird of the M1-projecting POm neurons responded to stimulating the inputs from S1 L5 (see Figure 3 in Mo and Sherman, 2019), suggesting that $S 1$ is not the main source of input to many of the POm neurons that project to M1. Furthermore, as the authors point out, even though most cells across all layers in M1 responded strongly to POm stimulation (see Figure 4 in Mo and Sherman, 2019), this activity was mediated by a larger set of POm cells than those receiving input from S1 L5. Nevertheless, the fact that S1 L5 $\rightarrow$ POm connections are relatively strong despite their sparsity, with unitary postsynaptic potentials from L5 axons able to elicit action potentials in POm cells (Mease et al., 2016c), supports the case for the $S 1$ $\mathrm{L} 5 \rightarrow \mathrm{POm} \rightarrow \mathrm{M} 1$ pathway being an efficient trans-thalamic communication channel.

A similar path between S1 and S2 through the POm was deemed critical for activation of S2 by stimulation of barrel field (S1 L5B) in vitro (Theyel et al., 2010). In that study, information transfer from S1 to S2 continued after permanent disruption of the direct corticocortical pro- jections, and was only interrupted by chemically induced thalamic inhibition. A similar experimental protocol could be applied to investigate the efficacy and the functional role of the $\mathrm{S} 1 \mathrm{~L} 5 \rightarrow \mathrm{POm} \rightarrow \mathrm{M} 1$ pathway in sensorimotor processing. Such an experiment would be valuable given that single-whisker-evoked responses in M1 were shown to depend on S1 activation (Ferezou et al., 2007).

Although the exact nature of information transmitted along the corticocortical and trans-thalamic S1 to M1 projections is unknown, Mo and Sherman (2019) suggest that these two routes carry different content because they originate from non-overlapping populations in S1 (Petrof et al., 2012). Furthermore, the corticothalamic cells in L5 projecting to POm also branch to subcortical motor centers (e.g., the brainstem; Krieger and Groh, 2015), indicating that these might transmit efference copies of motor-related instructions originating in S1 and forwarded to higher cortical centers (Sherman and Guillery, 2013). This hypothesis is supported by the fact that S1 was shown to participate in motor control and directly drive whisker retraction even after $\mathrm{M} 1$ inactivation (Matyas et al., 2010), suggesting that whisking-related instructions originate not only in M1 but also in S1.

However, information routed through the highly state-dependent POm is bound to undergo significant modulation as the nucleus can dynamically reconfigure its circuits depending on the arousal level (Sobolewski et al., 2015). During high alertness, POm combines not only descending cortical input, but also sensory information ascending via the paralemniscal pathway through the spinal trigeminal nucleus (mainly SP5i; Krieger and Groh, 2015). These inputs converge onto single thalamic cells, leading to timingsensitive nonlinear responses driven by coincident L5 and SP5i input, much like an "AND-gate" (Groh et al., 2014). Such convergence zones are spatially restricted, highlighting possible functional subdivisions within the nucleus (Mease et al., 2016b). Given that Mo and Sherman (2019) did not find any spatial localization of M1-projecting neurons in POm (their Figure $3 G$ ), these could overlap with the aforementioned convergence zones. If confirmed, then the trans-thalamic route might enable the integration of sensory input and cortical output in a time- and behavior-dependent manner en route to M1.

This pathway and its potential role in the temporally precise integration of sen- sory information become more interesting when one considers that transmission through the POm is potentially contingent on motor activity in M1 (Urbain and Deschênes, 2007). At rest, whisker responses in POm are gated through inhibitory projections from the zona incerta (ZI), an area also innervated by projections from M1. During active palpation, however, corticofugal messages from M1 modulating vibrissa motion can inhibit parts of ZI, thus creating a window of disinhibition in POm and enabling information flow. At the same time, POm neurons might relay signals back to $\mathrm{S} 1$, because some cells project to multiple sensory and motor cortical areas (Ohno et al., 2012). Reciprocal projections between POm and L5 of S1 have been suggested to participate in a cortico-thalamo-cortical loop that amplifies and temporally sustains certain relevant sensory content, similar to a vibrissal attention mechanism (Mease et al., 2016a). The POm and the newly mapped pathway are therefore strategically positioned to play a major role in sensorimotor processing, fusing both cortical and sensory input under top-down modulation from higher motor centers.

The findings of Mo and Sherman (2019) support the possibility that trans-thalamic pathways bridging functionally distinct cortical areas are a common theme in the mammalian brain. Although it is unclear whether such connections exist in other species, some tract-tracing studies in primates provide supportive evidence. In macaques, a potential route related to oculomotor processing might involve the mediodorsal nucleus, which receives input from area $7 \mathrm{~m}$ (medial posterior parietal cortex) and projects to area 8 (the frontal eye fields; Leichnetz, 2001). This putative pathway is also parallel to strong feedforward corticocortical connections (Markov et al., 2014), akin to the circuits observed in mice (Sherman and Guillery, 2013). In addition to mediating information flow from sensory to higher cortical areas, thalamic nuclei may also play an important role in executive control of motor behavior. McFarland and Haber (2002) proposed that direct signaling from prefrontal and higher motor centers to primary motor areas is complemented by a series of feedforward projections via the thalamus. This raises the possibility that thalamic pathways are involved in regulating information flow in both directions of the cortical hierarchy.

In conclusion, Mo and Sherman (2019) have confirmed the existence of a strong $\mathrm{S} 1 \mathrm{~L} 5 \rightarrow \mathrm{POm} \rightarrow \mathrm{M} 1$ pathway involving 
class 1 glutamatergic synapses, which is the first trans-thalamic connection revealed between functionally distinct cortical regions. Although this supports the case for such circuits being an integral link in cortical communication, further evidence is necessary to establish their generality beyond the sensorimotor areas and across species. Whereas the functional implications of this particular pathway remain unclear, it might play a key part in motor control, mediate sensory attention, or act as an integrator of peripheral and cortical signals. Narrowing down these potential roles is a challenging and exciting prospect that will require additional experimental work, including both quantitative thalamic connectivity data and targeted behavioral experiments on sensorimotor processing.

\section{References}

Ferezou I, Haiss F, Gentet LJ, Aronoff R, Weber B, Petersen CC (2007) Spatiotemporal dynamics of cortical sensorimotor integration in behaving mice. Neuron 56:907-923.

Groh A, Bokor H, Mease RA, Plattner VM, Hangya B, Stroh A, Deschênes M, Acsády L (2014) Convergence of cortical and sensory driver inputs on single thalamocortical cells. Cereb Cortex 24:3167-3179.

Guillery RW (1995) Anatomical evidence concerning the role of the thalamus in corticocortical communication: a brief review. J Anat 187:583-592.

Jaramillo J, Mejias JF, Wang XJ (2019) Engagement of pulvino-cortical feedforward and feedback pathways in cognitive computations. Neuron 101:321-336.e9.
Krieger P, Groh A (2015) Sensorimotor integration in the whisker system. New York: Springer.

Leichnetz GR (2001) Connections of the medial posterior parietal cortex (area $7 \mathrm{~m}$ ) in the monkey. Anatomical Record 263:215-236.

Markov NT, Ercsey-Ravasz MM, Ribeiro Gomes AR, Lamy C, Magrou L, Vezoli J, Misery P, Falchier A, Quilodran R, Gariel MA, Sallet J, Gamanut R, Huissoud C, Clavagnier S, Giroud P, Sappey-Marinier D, Barone P, Dehay C, Toroczkai Z, Knoblauch K, Van Essen DC, Kennedy H (2014) A weighted and directed interareal connectivity matrix for macaque cerebral cortex. Cereb Cortex 24:17-36.

Matyas F, Sreenivasan V, Marbach F, Wacongne C, Barsy B, Mateo C, Aronoff R, Petersen CC (2010) Motor control by sensory cortex. Science 330:1240-1243.

McFarland NR, Haber SN (2002) Thalamic relay nuclei of the basal ganglia form both reciprocal and nonreciprocal cortical connections, linking multiple frontal cortical areas. J Neurosci 22:8117-8132.

Mease RA, Metz M, Groh A (2016a) Cortical sensory responses are enhanced by the higherorder thalamus. Cell Rep 14:208-215.

Mease RA, Sumser A, Sakmann B, Groh A (2016b) Cortical dependence of whisker responses in posterior medial thalamus in vivo. Cereb Cortex 26:3534-3543.

Mease RA, Sumser A, Sakmann B, Groh A (2016c) Corticothalamic spike transfer via the L5B-POm pathway in vivo. Cereb Cortex 26:3461-3475.

Mo C, Sherman SM (2019) A sensorimotor pathway via higher-order thalamus. J Neurosci 39:692-704.

Nakajima M, Halassa MM (2017) Thalamic control of functional cortical connectivity. Curr Opin Neurobiol 44:127-131.

Ohno S, Kuramoto E, Furuta T, Hioki H, Tanaka YR, Fujiyama F, Sonomura T, Uemura M,
Sugiyama K, Kaneko T (2012) A morphological analysis of thalamocortical axon fibers of rat posterior thalamic nuclei: a single neuron tracing study with viral vectors. Cereb Cortex 22:2840-2857.

Petrof I, Viaene AN, Sherman SM (2012) Two populations of corticothalamic and interareal corticocortical cells in the subgranular layers of the mouse primary sensory cortices. J Comp Neurol 520:1678-1686.

Porter LL, White EL (1983) Afferent and efferent pathways of the vibrissal region of primary motor cortex in the mouse. J Comp Neurol 214:279-289.

Reichova I, Sherman SM (2004) Somatosensory corticothalamic projections: distinguishing drivers from modulators. J Neurophysiol 92:2185-2197.

Rovó Z, Ulbert I, Acsády L (2012) Drivers of the primate thalamus. J Neurosci 32:1789417908.

Sherman SM, Guillery RW (2013) Functional connections of cortical areas: a new view from the thalamus. Cambridge, MA: MIT.

Sherman SM, Guillery RW (2011) Distinct functions for direct and transthalamic corticocortical connections. J Neurophysiol 106: 1068-1077.

Sobolewski A, Kublik E, Swiejkowski DA, Kamiński J, Wróbel A (2015) Alertness opens the effective flow of sensory information through rat thalamic posterior nucleus. Eur J Neurosci 41:1321-1331.

Theyel BB, Llano DA, Sherman SM (2010) The corticothalamocortical circuit drives higherorder cortex in the mouse. Nat Neurosci 13:84-88.

Urbain N, Deschênes M (2007) Motor cortex gates vibrissal responses in a thalamocortical projection pathway. Neuron 56:714-725.

Ward LM (2013) The thalamus: gateway to the mind. Wiley Interdiscip Rev Cogn Sci 4:609-622. 